\title{
False negative results in glaucoma detection with Heidelberg Retina Tomograph II
}

\author{
Catherine Deghislage \\ Lidwine Van Malderen \\ Thierry G Zeyen \\ Department of Ophthalmology, \\ University Hospitals, Leuven, B-3000, \\ Belgium
}

\begin{abstract}
Purpose: To evaluate the rate of false negative results with the Heidelberg Retina Tomograph (HRT II) in a glaucoma practice.

Design: Cross-sectional study.

Methods: We analyzed the HRTs taken between October 2002 and October 2003 in our glaucoma clinic, and selected the patients who had a good quality image (SD $<40 \mu)$ with a normal Moorfield's Regression Analysis (MRA). A masked independent observer classified those patients as normal, glaucoma suspect, or glaucomatous on the basis of optic disc stereo photos (ODP) and at least 2 consecutive reliable automated perimetries. The diagnosis of glaucoma was based on a glaucomatous optic disc with a congruent, reproducible visual field defect.

Results: Four hundred and fifty patients who had undergone an HRT examination were analyzed. One hundred and nine patients had an HRT classified as normal on the MRA, and a good quality image. Fifteen of those 109 patients (13.7\%) were classified as glaucomatous on the basis of an abnormal ODP with corresponding visual field defect. Seven (6.4\%) patients were classified as glaucoma suspect.
\end{abstract}

Conclusion: Fourteen percent of glaucoma patients with glaucoma remained undetected with the HRT II Moorfield's regression analysis as a sole means to detect glaucoma.

Keywords: glaucoma detection, false negative results, Heidelberg Retina Tomograph II

\section{Introduction}

Changes in the structural appearance of the optic nerve head and retinal nerve fibre layer (RNFL) often precede the development of visual field loss in glaucoma. Therefore detection of optic nerve head and RNFL damage is crucial for the diagnosis of glaucoma in its early stages (Zeyen et al 1993; Sanchez-Galeana et al 2001; Kass et al 2002).

Until recently, structural evaluation of glaucomatous damage has been subjective, with primarily qualitative descriptions of changes. In a clinical setting, ophthalmoscopy is the most frequently used method, but it has only a moderate interobserver agreement (Tielsch et al 1988; Lichter 1997).

Stereoscopic biomicroscopy and optic disc photography are widely used, but are also associated with a considerable intra- and interobserver variability, mostly based on the examiner's experience (Ikram et al 2002; Miglior et al 2002).

New methods such as confocal scanning laser ophthalmoscopy, scanning laser polarimetry, and optical coherence tomography have been developed in the last decade to provide real-time, objective and quantitative information of the optic nerve head and RNFL.

Confocal scanning laser ophthalmoscopy is a technology that uses the different properties of light and characteristics of retinal tissue to obtain the necessary measurements. The Heidelberg Retina Tomograph (HRT II, Heidelberg engineering, Heidelberg, Germany) has been widely investigated as a research tool in the imaging of the optic nerve head and its method of analysis has been described elsewhere (Kamal et al 1999; Medeiros et al 2004). 
It has been shown to detect glaucomatous optic nerve damage with clinically significant sensitivity and specificity (Wollstein et al 1998; Gordon et al 1999; Sanchez-Galeana et al 2001). The HRT classification of the optic disk, that was used, is based on the Moorfield's Regression Analysis (MRA), one of the most used analysis tools included in the HRT software. The MRA classification takes into account the global and sectorial rim area corrected for the global and sectorial disk area. Furthermore, it uses the classification of normal, borderline and outside normal limits (Miglior et al 2003).

The HRT could be used for glaucoma detection provided the rate of false negative (FN) results (a negative test result while disease is present) is not too high. In this study we sought to determine the rate of FN results with the HRT II in a glaucoma practice.

\section{Design}

Cross-sectional study performed in one glaucoma clinic.

\section{Materials and Methods}

We analysed all HRT examinations consecutively taken between October 2002 and October 2003 in our glaucoma clinic and selected the patients who had a good quality image (Standard deviation $(\mathrm{SD})<40 \mu$ ) with a normal MRA. All images were obtained by the same operator. Only one randomly selected eye per patient was included in our study group.

An experienced, masked independent observer classified patients as normal, glaucoma suspect, or glaucomatous on the basis of optic disc stereo photos (ODP) and at least 2 consecutive reliable automated perimetries taken within 6 months of the ODPs. The diagnosis of glaucoma was based on a glaucomatous optic disc with a corresponding, reproducible visual field defect.

Our study was not approved by an ethic committee.

\section{Instrumentation}

\section{The HRT II}

The HRT II is a confocal scanning laser ophthalmoscope that uses a $670 \mathrm{~nm}$ wavelength diode laser and therefore does not require dilation. The laser beam is scanned across the retina in $\mathrm{x}$ and $\mathrm{y}$ directions. This confocal scanning technique uses the pinhole principle focusing for both incoming and outgoing laser beam. Thus, only light reflected at or near the adjusted focal plane is detected and contributes to the image. The topographic image is derived from multiple optical sections at consecutive focal depth planes. Each image consists of numerous pixels, with each pixel corresponding to the retinal height at its location. By color coding the area of depression and elevation, the HRT II provides a 2-dimensional representation. Tracing the contour line was the only subjective step and was performed masked from diagnosis. After definition of the optic contour line, the 14 stereometric parameters, a comparison to previous examinations and to the normative database are presented (Zeyen et al 1993).

To properly classify the optic disk, the HRT II uses the MRA approach. It has the advantage of adjusting the global and sectorial rim areas for disc size and age to improve specificity and to allow for the assessment of regional damage. Regional damage is assessed by dividing the optic nerve head into 6 sectors (nasal, supranasal, supratemporal, temporal, infratemporal, and inferonasal). Compared to those in a normal database using MRA, these sectors are classified into 1 of 3 categories (within normal limits, borderline, or outside normal limits) (Miglior et al 2003).

Briefly, this classification is performed as follows: the rim and disc area for each sector are compared with those in a normal database and the sectors are then classified depending on the patient's age and the overall size of the optic disc. The analysis provides a predicted value and an actual value for the rim area of each of the 6 sectors. If the percentage of the rim area is larger than or equal to the $95 \%$ age-dependent confidence interval/limit, then the sector is classified as being within the normal limits. If the percentage of rim area is between $95 \%$ and $99.9 \%$ confidence interval, then the respective sector is classified as borderline. Finally, if this percentage is lower than the $99.9 \%$ confidence limit, the sector can be classified as outside the normal limits.

Furthermore, the MRA provides results for the global rim area as well as a final classification. A normal classification requires the MRA of all sectors and the global rim area to be within the normal limits (Mikelberg et al 1995; Wollstein et al 1998).

\section{Visual field testing}

Visual field testing was performed with the Humphrey Field Analyser (HFA), program 24-2, SITA standard strategy or the Octopus 301, program G1 $\left(30^{\circ}\right)$, dynamic strategy.

All patients required at least two consecutive reliable visual fields tests performed with the same perimeter (and strategy). Reliability criteria applied were fixation losses less than 30\% (Heijl- Krakau method) for HFA and false-positive responses less than $20 \%$, and false-negative responses less than 30\% for both HFA and Octopus(Zeyen 1997).

Glaucomatous visual field loss was considered significant when there was an abnormal Glaucoma Hemifield Test on two 
consecutive examinations (for the HFA) or in the presence of 3 identical contiguous test locations, not contiguous with the blind spot, on two consecutive examinations, with $\mathrm{p}<5 \%$ probability of being normal (one of which should have $\mathrm{p}<1 \%$ ) for both HFA and Octopus (Flammer 1986; Asmann et al 1992; EGS 2004).

\section{Stereoscopic colour optic nerve head photographs}

In one visit, patients underwent HRT imaging and had standard stereoscopic colour optic nerve head photographs taken. These were taken by the same operator with the Kowa Mydriatic camera at a $20^{\circ}$ setting after maximal dilatation. Two sequential photographs were taken per eye, with a lateral shift in camera position to obtain a stereo effect. All photographs were analyzed by one experienced clinician using a stereoscopic viewer. The clinician was masked from the patient's data. Definition of glaucomatous damage was based on focal or diffuse narrowing of the neuroretinal rim (EGP 2003).

The diagnosis of glaucoma was based on a glaucomatous optic disc with a corresponding, reproducible visual field defect. HRT examinations, ODPs and visual field examination were performed within a maximum interval of six months.

Glaucoma suspects were defined as patients with normal optic disk and abnormal visual field and patients with abnormal optic disk compatible with glaucoma and a normal visual field.

\section{Results}

Four hundred and fifty HRT examinations of good quality, taken between October 2002- October 2003, were analysed. After the exclusion of eyes classified as abnormal on the MRA, 109 (65 women, 44 men) patients were included for analysis.

The median age was 61.45 years (range $29-83$ years) (Table 1).

Fifteen (13.7\%) of those 109 patients were diagnosed as glaucomatous on the basis of an abnormal ODP with matching visual field defect. Results were summarized in a flow diagram (Figure 1). Figure 2 shows one of the 15 patients. Of those 15 patients 9 had focal rim loss and 6 had diffuse rim loss on optic disc stereo photos. Seven (6.4\%) patients were diagnosed as glaucoma suspect (Figure 2).

Octopus visual field testing was performed in 52 of the 109 eyes, HFA examinations were performed in 61 of the 109 eyes. The severity of visual field loss in the FN group was characterized by a mean deviation of -6.0 (range -0.11
Table I Summary of patient characteristics

109 patients included for analysis

\begin{tabular}{ll}
\hline Sex: & 65 \\
-Women & 44 \\
-Men & 61.45 (range 29-83) years \\
Mean Age: & \\
\hline
\end{tabular}

to -16.9$)$. In the glaucoma suspect group the mean deviation was -6.1 (range 0.91 to -18.15 ).

\section{Discussion}

In patients with glaucoma, optic nerve head modifications often precede glaucomatous visual field alterations. To detect glaucoma it seems therefore appropriate to use digitalized imaging capable of detecting glaucomatous optic disc alterations in early stages.

In this study we investigated the diagnostic accuracy of the HRT II, particularly in terms of glaucoma detection.

Sensitivities and specificities have commonly been reported as reliable parameters to evaluate the diagnostic precision of devices in studies. However, their clinical utility is limited.

They reflect the probability that a particular test result is positive or negative given the presence (sensitivity) or absence (specificity) of disease (Jaeschke et al 1994; Gallagher 2003; Moons et al 2003). We preferred to look at the rate of FN results relevant in daily practice. In glaucoma, a disease with a relatively low incidence in which symptoms appear late, it is important not to miss the diagnosis. Hence a low $\mathrm{FN}$ rate is required.

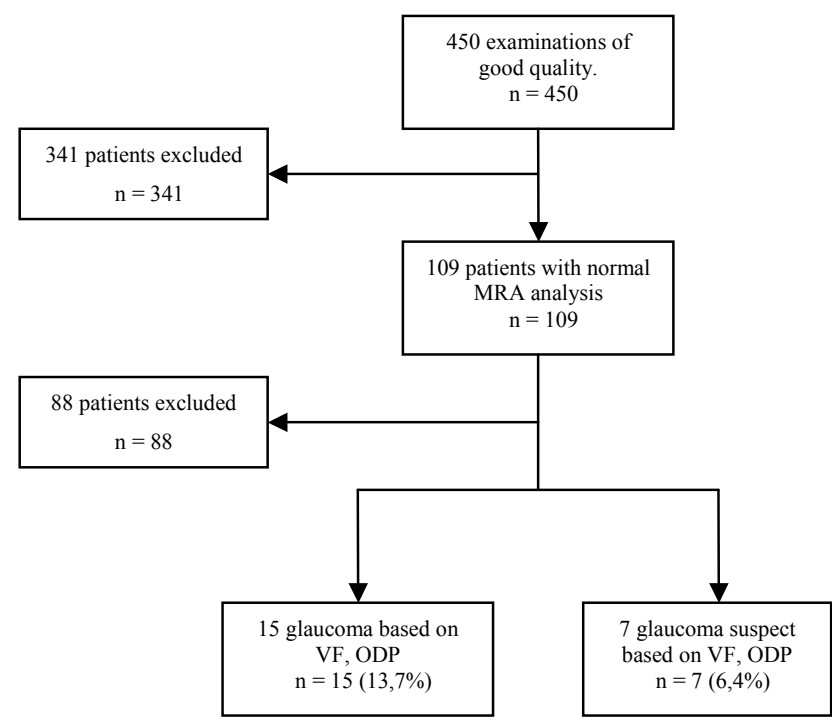

Figure I Results. 


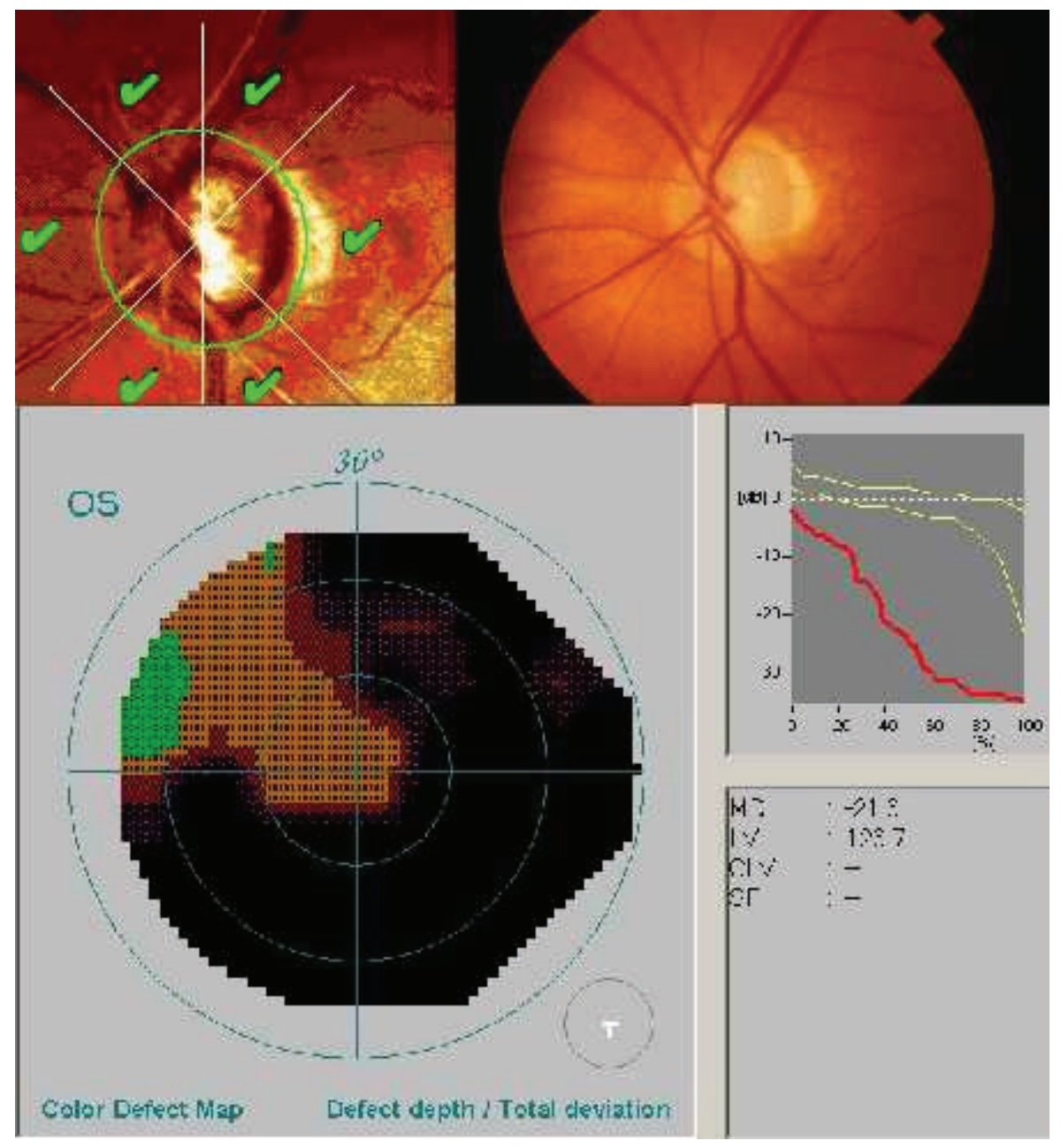

Figure 2 Example of false negative result. HRT with normal MRA analysis, but glaucomatous cupping on ODP with corresponding visual field defect on automated perimetry. Abbreviations: HRT, Heidelberg Retina Tomography; MRA, Moorfields regression analysis; ODP, Optic Disc Photograph.

In the literature the rate of $\mathrm{FN}$ results varies between 14 and $26 \%$. Wollstein, Mikelberg, Miglior, and their colleagues reported FN rates of $15.7 \%, 16 \%$, and $26 \%$, respectively when glaucoma suspects were excluded (Mikelberg et al 1995; Wollstein et al 1998; Miglior et al 2003). Kesen and colleagues (2002) and Ford and colleagues (2003) found FN rates of $14 \%$ and $22 \%$, respectively when glaucoma suspects were counted as positive results. Those rates are in the same range of the FN results of our study: $13.7 \%$ when glaucoma suspects are excluded, and $20.1 \%$ when glaucoma suspects are included.

Most of our glaucoma patients with FN results (9/15) had localized rim loss on stereo photos. It may be that those patients would have been detected with the new software for the HRT, Advanced Glaucoma Analysis 3.0, which includes a larger normative database and new data analysis tools such as the Glaucoma Probability Score (GPS).

There are some weaknesses in the methodology of the present study. Firstly, only two consecutive visual fields were considered for the diagnosis of glaucoma whereas for most randomized clinical trials, as the Ocular Hypertension Treatment Study (Greaney et al 2002) or the European Glaucoma Prevention Study (2005), at least three consecutive visual field examinations were required. We might therefore have overestimated the number of glaucoma patients.

The second weakness in our study is that it was conducted in a referral centre, with a large number of glaucoma patients. In general practice the number of glaucoma patients would be lower and therefore the rate of false negative results might be lower as well.

Finally, we did not consider the rate of false positive results which reflects over-screening and leads to an unnecessary work-up to rule out the diagnosis.

Although one single test does not presently ideally identify glaucomatous damage, disc examination is probably the best single test for glaucoma detection. Our relatively high rate of FN results with the HRT II MRA to screen for glaucoma 
can probably be reduced by combining this examination with a functional detection test that is easy to perform and can detect abnormalities in an early stage, such as the Frequency Doubling Technology (Brusini et al 2006).

In conclusion, $13.7 \%$ of glaucoma patients remain undetected with the HRT II MRA alone to detect glaucoma.

\section{References}

Asmann P, Heijl A. 1992. Glaucoma hemifield test: Automated visual field evaluation. Arch Ophthalmol, 110:812-19.

Brusini P, Salvetat ML, Zeppieri M, et al. 2006. Frequency doubling technology perimetry with the Humphrey Matrix 30-2 test. J Glaucoma, 15:77-83.

[EGS] European Glaucoma Society. 2004. Terminology and Guidelines for Glaucoma. 2nd Ed [online]. Accessed on 9 January, 2007. URL: http://www.eugs.org/fullbook.asp.

[EGP] European Glaucoma Prevention Study Group. 2003. Reproducibility of evaluation of optic disc change for glaucoma with the stereo optic disc photographs. Ophthalmology, 110:340-4.

[EGP] European Glaucoma Prevention Study Group. 2005. Results of the European Glaucoma Prevention Study. Ophthalmology, 112: 366-375.

Flammer J. 1986. The concept of visual field indices. Graefe's Arch Clin Exp Ophthalmol, 224:289-392.

Ford B, Artes FH, McCormick TA, et al. 2003. Comparison of data analysis tools for detection of glaucoma with the Heidelberg retina tomograph. Ophthalmology, 110:1145-50.

Gallagher EJ. 2003. Evidence-based emergency medicine/editorial: the problem with sensitivity and specificity. Ann Emerg Med, 42:298-303.

Gordon MO, Kass MA; for the Ocular Hypertension Treatment Study Group. 1999. The Ocular Hypertension Treatment Study. Design and baseline description of the participants. Arch Ophthalmol, 117:573-83.

Greaney MJ, Hoffman DC, Garway-Heath DF, et al. 2002. Comparison of optic nerve imaging methods to distinguish normal eyes from those with glaucoma. Invest Ophthalmol Vis Sci, 43:140-5.

Ikram MK, Borger PH, Assink JM, et al. 2002. Comparing Ophthalmoscopy Slide viewing, and semiautomated Systems in Optic Disc Morphometry. Am J Ophthalmol, 109:486-93.

Jaeschke R, Guyatt GH, Sackett DL; for the Evidence-Based Medicine Working Group. 1994. User's guides to the medical literature, III: how to use an article about a diagnostic test, B: what are the results and will they help me in caring for my patients? JAMA, 271:703-7.
Kamal DS, Viswanathan AC, Garway-Heath DF, et al. 1999. Detection of optic disc change with the Heidelberg Retina Tomograph before confirmed visual field change in ocular hypertensives converting to early glaucoma. Br J Ophthalmol, 83:290-4.

Kass MA, Heuer DK, Higginbotham EJ, et al. 2002. The ocular Hypertension Treatment Study: a randomised trial determines that topical ocular hypotensive medication delays or prevents the onset of primary openangle glaucoma. Arch Ophthalmol, 120:701-13.

Kesen MR, Speath GL, Henderer JD, et al. 2002. The Heidelberg retina tomograph vs Clinical impression in the diagnosis of Glaucoma. Am J Glaucoma, 133:613-16.

Lichter PR. 1997. Variability of expert observers in evaluating the optic disc. Trans Am Ophthalmol Soc, 74:532-72.

Medeiros FA, Zangwill LM, Bowd C, et al. 2004. Comparison of the GDXVCC scanning laser polarimeter, HRT II confocal scanning laser ophthalmoscope, and stratus OCT optical coherence tomograph for the detection of glaucoma. Arch Ophthalmol, 122:827-37.

Miglior S, Albé E, Guareschi M, et al. 2002. Intraobserver and interobserver reproducibility in the evaluation of optic disc stereometric parameters by Heidelberg Retina Tomograph. Ophthalmology, 109:1072-7.

Miglior S, Guareschi M, Albé E, et al. 2003. Detection of glaucomatous visual field changes using the Moorfield's regression analysis of the Heidelberg retina tomograph. Am J Ophthalmol, 136:26-33.

Mikelberg FS, Parfitt CM, Swindale NV, et al. 1995. Ability of the Heidelberg retina tomograph to detect early glaucomatous visual field loss. J Glaucoma, 4:242-7.

Moons KG, Harrell FE. 2003. Sensitivity and specificity should be deemphasized in diagnostic accuracy studies. Acad Radio, 370-672.

Sanchez-Galeana C, Bowd C, Blumenthal EZ, et al. 2001. Using optical imaging summary to detect glaucoma. Ophthalmology, 108:1812-18.

Tielsch JM, Katz J, Quiqley HA, et al. 1988. Intraobserver and interobserver agreement in measurement of optic disc characteristics. Ophthalmology, 95:350-6.

Wollstein G, Garway-Heath DF, Hitchings RA. 1998. Identification of early glaucoma cases with the scanning laser ophthalmoscope. Ophthalmology, 105:1557-63.

Zeyen TG, Caprioli J. 1993. Progression of disc and field damage in early glaucoma. Arch Ophthalmol, 111:162-5.

Zeyen TG. 1997. Interpretation of automated perimetry. Bull Soc Belge Ophthalmol, 267:191-7. 
